\title{
Referentes curriculares con incorporación tecnológica para la formación de profesores de matemática en y para la diversidad
}

\author{
Olga Lucía León Corredor ${ }^{1}$ \\ A mis colegas y amigos: \\ Mariana, Edith, Rosa María, Napoleón, Marlón, William, Diana, Martha, \\ Mónica, Cecilia, Jorge, Alejandro, Andrea y Jaime de la Comunidad ALTER-NATIVA de Matemáticas.
}

\section{Resumen}

El proyecto ALTER-NATIVA es el contexto de producción del libro "Referentes Curriculares con Incorporación de Tecnologías para la Formación del Profesorado de Matemáticas en y para la Diversidad”. La Comunidad ALTER-NATIVA de Matemáticas (CAM), fue conformada por profesores, estudiantes para profesor, investigadores y poblaciones sordas y ciegas, pertenecientes a la Universidades de: Regiones Autónomas de la Costa Caribe Nicaragüense URACCAN de Nicaragua, Pedagógica Nacional de México UPN, "Distrital Francisco José de Caldas" UDFJC de Colombia y al Instituto Nacional para Sordos INSOR.

Palabras clave: Referente curricular; Formación de profesores; Tecnologías educativas; Didáctica Matemática; Diversidad.

\section{Summary}

The ALTER-NATIVA project is based on the context of the production of the book entitled "Curricular reference with the incorporation of technology for mathematics teachers training in/and for diversity". The ALTER-NATIVA Community of Mathematics (CAM), was made up of teachers, student teachers, researchers and deaf and blind populations belonging to: the University of the Autonomous Regions of the Caribbean Coast of Nicaragua (URACCAN), the National Pedagogical of Mexico-UPN, the Francisco Jose de Caldas District-UDFJC of Colombia and the National Institute for the Deaf-INSOR.

Keywords: Curricular reference; Teachers training; Educational technologies; Mathematics Teaching; Diversity.

\section{Introducción}

Los "Referentes Curriculares con Incorporación de Tecnologías para la Formación del Profesorado de Matemáticas en y para la Diversidad", son el resultado del reconocimiento de cinco aspectos fundamentales: las múltiples experiencias con lo matemático

1 Doctora en Educación, Profesora e Investigadora de la Universidad Distrital "Francisco José de Caldas", Colombia. Email: oleoncorredor@gmail.com 
y su didáctica; las múltiples representaciones de lo matemático y de su didáctica; los múltiples tipos de interacción en los ambientes de aprendizaje; las poblaciones en vulnerabilidad educativa por sus condiciones sensoriales, étnicas o económicas; y una metodología de interacción y desarrollo definida por las comunidades de práctica.

La organización de la obra en tres campos de formación de profesores - la educación, la pedagogía y la didáctica de la matemática - permitió presentar de manera específica, explicaciones teóricas atendiendo a las condiciones pedagógicas y didácticas en Educación Matemática y a sus relaciones con las diversas poblaciones involucradas en ALTER-NATIVA, se hace además una relación con las tecnologías tanto TIC como con otras diferentes, pero necesarias y útiles en el aprendizaje de las matemáticas.

\section{Por lo anterior, la obra Referentes ${ }^{2}$ presenta una estructura de contenidos que ofrece:}

- El Contexto de producción de los Referentes Curriculares. En donde se identifican las problemáticas transversales en el contexto de la formación de profesores de matemáticas, para atender poblaciones diversas.

- La Estrategia de trabajo para la construcción consensuada de los Referentes Curriculares. Se describe el papel y el funcionamiento de la Comunidad de Práctica como metodología de trabajo, en la construcción de los Referentes Curriculares.

- El marco de análisis de las relaciones pedagógicas y didácticas en Educación Matemática. Involucra las tecnologías empleadas en el contexto de la enseñanza y el aprendizaje de las matemáticas, las formas en que se manifiesta el compromiso de formar profesores de matemáticas en y para la diversidad.

- La reflexión y el consenso sobre la didáctica de las matemáticas. Explicaciones teóricas, atendiendo a las condiciones pedagógicas y didácticas, sobre la cantidad, la magnitud y la forma, como campos de formación matemática de necesaria presencia en los currículos de matemáticas y a sus relaciones con las diversas poblaciones involucradas EN ALTER-NATIVA y con las tecnologías tanto TIC como diferentes a éstas. En este sentido, en los Referentes se identifican y construyen: i) Variantes e invariantes curriculares: ejes transversales en la formación de profesores, campos estructurantes específicos para la formación de profesores en matemáticas; ii) Un conjunto de veintiún (21) Referentes Curriculares que articulan aspectos profesionales, pedagógicos y didácticos en la formación de profesores de Matemáticas.

- Orientaciones para la operacionalización y/o utilización de los referentes. Se proponen orientaciones para tres tipos de instituciones y destinatarios del sistema educativo: los estamentos que crean y regulan las políticas educativas,

2 En adelante la palabra Referentes es usada para aludir a "Referentes curriculares con incorporación tecnológica para la formación del profesorado de matemáticas eny para la diversidad". 
las instituciones de Educación Superior, particularmente las facultades y programas de pregrado que forman profesores en las tres áreas mencionadas y la escuela y específicamente los profesores en formación y en ejercicio.

La organización de la obra en tres campos de formación de profesores: la educación, la pedagogía y la didáctica de la matemática, responde la necesidad de una formación de profesores de matemáticas, que responda a las exigencias de no marginación de poblaciones de la Educación Matemática en América Latina. Se trata de formar profesores para que lo humano y la humanidad son ineludibles en la Educación Matemática que ellos promueven. Es inevitable la reflexión sobre las tecnologías tanto TIC como diferentes a éstas, pero necesarias y útiles en el aprendizaje de las matemáticas.

\section{La formación de profesores de Matemática y el acceso a la Educación Superior de poblaciones vulnerables}

Le vigencia en el 2015 de los Referentes publicados en el 2013, en la formación de profesores y el acceso a la Educación Superior de las poblaciones vulnerables, se puede fundamentar con estudios actualizados de la situación de la educación en ALC en 2015. En los Referentes se manifiesta el propósito de desarrollar una identidad de profesor de matemáticas en el ambiente de América Latina y el Caribe (ALC), con el conocimiento de su región y de las posibilidades de coexistencia de esta región con las demás regiones del mundo, es explicito el reconocimiento de que aunque las políticas públicas de los países de América Latina y el Caribe en la década pasada -y por supuesto, en la presente- han proclamado el propósito de luchar contra la desigualdad y promover la igualdad de oportunidades con equidad, la situación de no acceso a la Educación Superior de la mayoría de jóvenes de ALC se mantiene o se aumenta..

Los siguientes tópicos son objeto de profundización en los Referentes en lo que refiere a desigualdad educativa y sus manifestaciones en América Latina y el Caribe:

- Disparidad en logros de aprendizaje y la desigualdad en el acceso a oportunidades educativas de calidad.

- El bajo nivel de acceso a las Instituciones de Educación Superior (IES).

- Políticas educativas de la región, cobertura calidad en inclusión.

- Recursos y acceso a Internet.

- Profesores de América Latina y el Caribe y su formación.

Se destaca que el modelo de formación de profesores más extendido es el de la poca formación didáctica y pedagógica en prácticas con estudiantes en situación de vulnerabilidad, con la escasa formación política y tecnológica para desplegar aprendizajes matemáticos en niñas, niños y jóvenes pertenecientes a poblaciones vulnerables. 
Estudios posteriores a la publicación de Referentes indican que sigue siendo vigente el aspecto que motivo a las universidades de ALTER-NATIVA construir los referentes (Figura No. 1)

\section{América Latina (18 países) ${ }^{\text {a }}$ situación de actividad de jóvenes de 15 a 29 años que no estudian y no tienen un empleo remunerado, alrededor de 2012}

(En porcentajes)

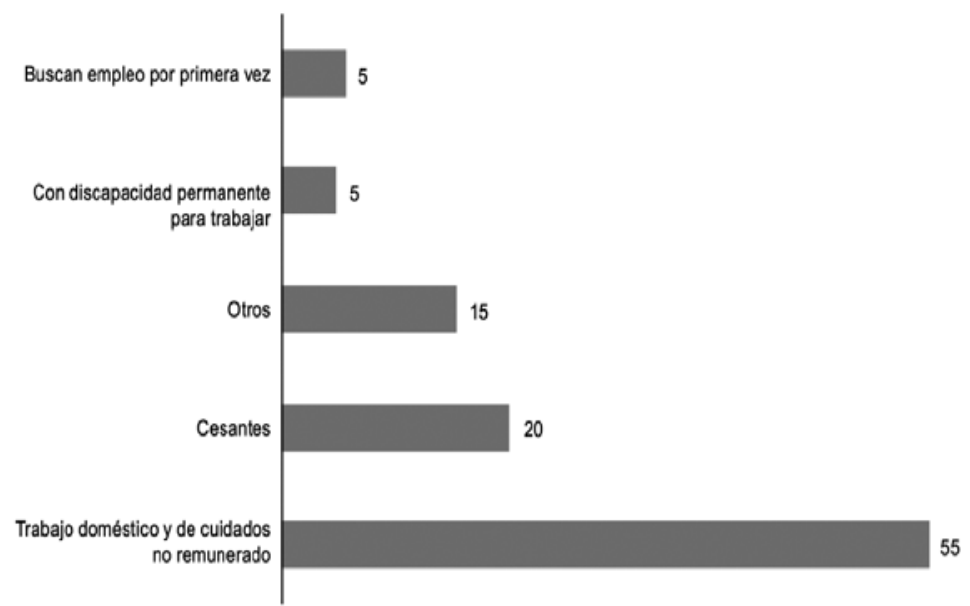

Comisión Económica para América Latina y el Caribe (CEPAL), sobre la base de tabulaciones especiales de las encuestas de hogares de los r Argentina (zonas urbanas), Bolivia (Estado Plurinacional de), Brasil, Chile, Colombia, Costa Rica, Ecuador, El Salvador, Guatemala, Honduras, $\mathrm{N}$ Panamá, Paraguay, Perú, República Dominicana, Uruguay y Venezuela (República Bolivariana de).

Figura No. 1. Situación de jóvenes de 15 -29 años, 2012

En los datos que se reportan en Referentes, 31.4\% de los latinoamericanos vive en condiciones de pobreza, el 12.3\% de estos son indigentes o están en extrema pobreza. En las publicaciones más recientes de la CEPAL se proyectaba para 2014 un leve incremento de la tasa de indigencia, que sería de alrededor del 12\%, lo que significaría "no solo un retroceso de esta tasa hasta los niveles observados a principios de la década, sino también un crecimiento apreciable de la cantidad de personas afectadas por esta situación, debido al crecimiento demográfico" (CEPAL, 2014, pág. 16).
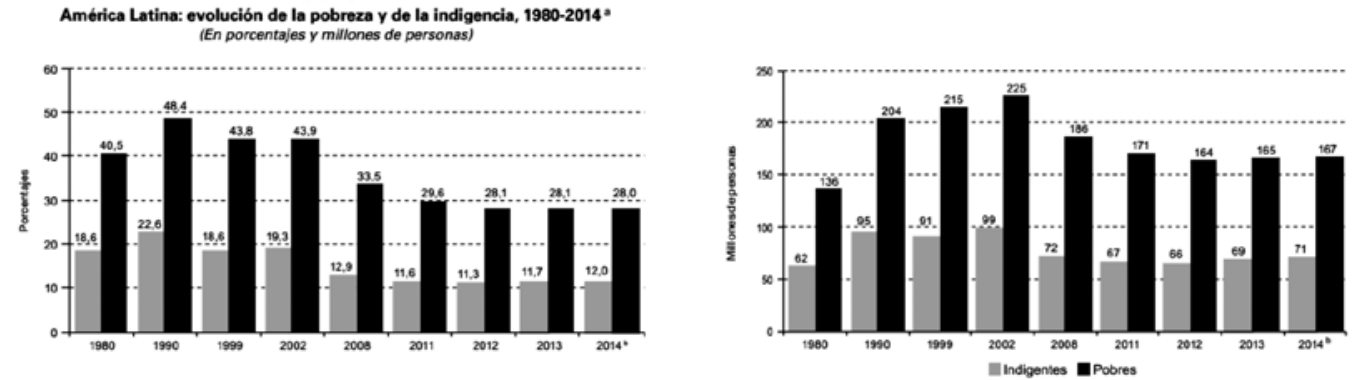

Figura No. 2. Evolución de la pobreza y la indigencia 1980-2014. Fuente (EPAL 2014, p.16) 
En los Referentes también se destaca que en la primera década de este milenio la Situación de la Educación de los Indígenas en ALC requería grandes cambios en los sistemas educativos:

Finalmente, los resultados en la pruebas de calidad, en especial en el estudio Serce (2008), muestran que hay una gran proporción de alumnos que no alcanzan el nivel mínimo de aprendizaje esperado en las pruebas de matemáticas y lenguaje, proporción que aumenta cuando se tiene en cuenta a la población indígena, lo que indica, una vez más, que en las condiciones actuales, el acceso a la educación no garantiza la equidad educativa, ya sea a causa de las condiciones de enseñanza, de aprendizaje o de realización de las pruebas a las que los sistemas educativos de América Latina y el Caribe someten a esta población de estudiantes (25).

El fenómeno de marginación se extiende con mayor efecto en poblaciones con alguna situación de discapacidad:

En Colombia el porcentaje de personas ciegas que no saben leer ni escribir se acerca al 34\%, únicamente el $43 \%$ de esta población entre 6 y 44 años asiste a la escuela y solo el o.3\% obtiene un título universitario. En México el 1.36\% de los escolares está en alguna situación de discapacidad; de estos, cerca de $65 \%$ tiene problemas visuales y aquellos en situación de discapacidad motora o auditiva representan menos del 20\%. En Nicaragua, por su parte, para 2004 se encontró que tan solo 1 400 estudiantes con necesidades educativas especiales eran atendidos en Centros Educativos Regulares y que el MINED (Dirección de Educación Especial del Ministerio de Educación) atiende a 5,515 niños en diferentes situaciones de discapacidad en Centros de Educación Especial, identifica debilidades y fortalezas en las habilidades de niños indígenas, su desarrollo y situación frente a las exigencias y estándares de la cultura dominante (26).

El análisis que la comunidad CAM hizo de la situación de marginación de poblaciones de los sistemas educativos se completó con una revisión documental de la situación de formación de profesores de matemáticas:

Cobra especial interés lo que Shulman (1986) denominó conocimiento de la materia a enseñar y que otros han llamado matemáticas para enseñar o conocimiento matemático para enseñar. Ball, Thames y Phelps (2008) caracterizan el conocimiento del profesor de matemáticas como un conocimiento de carácter práctico profesional; conjugan el conocimiento de la Matemática y el contenido pedagógico (Shulman, 1986), y lo denominan "conocimiento matemático para la enseñanza», como saber necesario para realizar la práctica de enseñar matemáticas. Desde una formación 
matemática en y para la diversidad, es necesario contemplar, además, el conocimiento del entorno cultural del aula (49).

\section{Caracterización y metodología de construcción de los referentes curriculares}

Un referente curricular para la formación de profesores de matemáticas es un sistema compuesto por dos dimensiones:

- La enunciativa, que lo constituye como una expresión que considera, en sí misma, la articulación de un sistema de relaciones políticas, éticas, geográficas, económicas, científicas, tecnológicas, educativas, pedagógicas y didácticas, para ser punto de referencia para la formación de profesores de matemáticas de poblaciones que coexisten en la diversidad y fomentan la coexistencia de la diversidad en y desde América Latina y el Caribe.

- La operativa, que lo considera como una forma de actuar en la formación de profesores, que se constituye en ejemplo a seguir.

Las dos dimensiones anteriores se articularon para dar forma en lo enunciativo a un conjunto de referentes que tuvieran fuerza de realización y en los operativo a un sistema didáctico compuesto por unas orientaciones para la incorporación de tecnología en la enseñanza de las matemáticas y un objeto virtual de aprendizaje.

Los siguientes aspectos fueron considerados para organizar la metodología de construcción de los referentes:

- El aspecto de constituir comunidades de prácticas con profesores, estudiantes, investigadores y grupos sociales marginados de la Educación Matemática: "son indicadores de la existencia de una comunidad de práctica los siguientes factores: conformación de una identidad de participación basada en relaciones mutuas; decantación de un propósito común continuamente abordado; configuración de un repertorio común"(28).

- La organización y aplicación de una metodología de construcción coherente con la dinámica de una comunidad de práctica.

- La identificación de variantes e invariantes curriculares para la organización de un referente curricular. 
La siguiente figura presenta el proceso seguido:

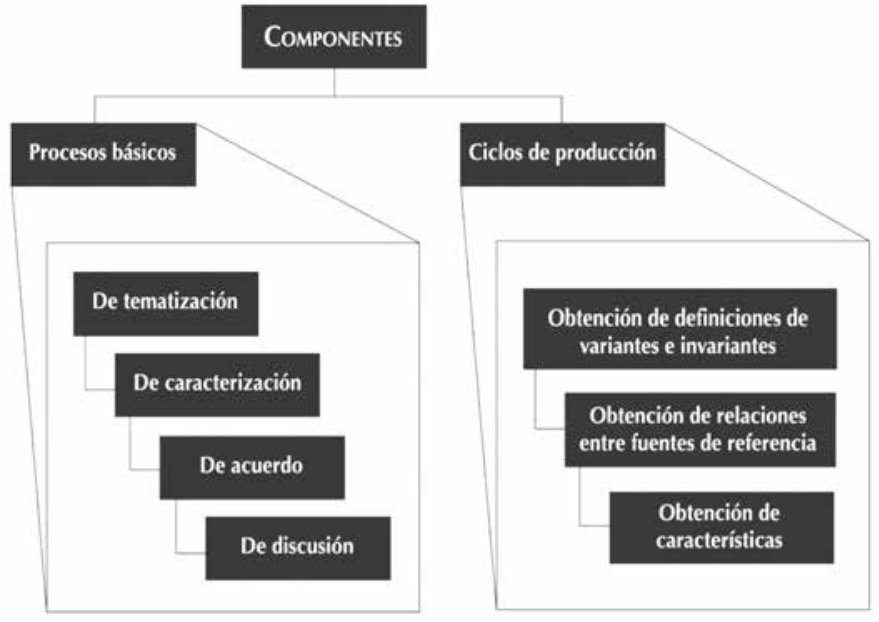

Figura No. 3. Estructura para la producción de referentes. Fuente (León et. al , 2014 p.28)

Las etapas de formulación de los referentes después de obtenidas las variantes e invariantes fueron: Agrupación de variantes e invariantes por categorías; formulación de referentes; validación interna de los referentes y validación externa y en escenarios naturales.

\section{Estructura de los referentes}

Este aspecto se profundiza ampliamente en el capítulo tres de los Referentes, que identifica como ejes curriculares transversales: La diversidad y la tecnología como ejes que impactan en la constitución de ambientes pedagógicos y didácticos; las didácticas específicas como ejes que proponen campos de formación específicos en la constitución del sujeto profesor de Ciencia Matemática: y la formación de profesores como el gran eje curricular articulador de la educación como profesión.

\section{Ejes y actividades para formar y para aprender a enseñar}

En los referentes se plantea una caracterización de formación como proceso y como resultado:

En tanto proceso, la formación es una fase amplia que involucra todas las transformaciones de enculturación que una generación desarrolla hacia las generaciones siguientes, desde los diferentes escenarios de interacción y de conformación de grupos de los que dispone. Los procesos formativos están presentes a lo largo de toda la vida para cada generación; «se dan en todo tiempo y lugar cuando cada cultura 
inicia a sus niños, niñas y jóvenes en el mundo de la vida como horizonte de todas las prácticas culturales» (Vasco, et al., 2008, p. 113).

La formación, en tanto resultado, se considera el efecto de una práctica social como, por ejemplo, la finalización del aprendizaje de un oficio (León, et al., 2014, p.67)

Generalmente el reconocimiento social de la formación como un producto académico es un documento que se denomina "Diploma". En el caso de la formación de profesores las universidades o Escuelas Normales Superiores (según sea el país) otorgan título de profesional en educación.

Los Referentes reconocen la formación como el macro proceso y la educación como un tipo de formación asumida por una institución (escuelas, universidades) o por grupos sociales con reconocimiento institucional (iglesias, clubes, comunidades que constituyen organismos gubernamentales y no gubernamentales).

La Educación la Pedagogía y la Didáctica son ejes de formación de profesores en tanto que:

- La educación es el campo profesional del educador matemático con una práctica social que articula la acción profesional de todos los educadores.

- La pedagogía, como saber teórico práctico generado por un ejercicio interdisciplinario y transdisciplinario, se constituye en un campo de conocimiento sistemático propio de los docentes. Desde una perspectiva de formación de profesores para la diversidad, se retoma la enseñanza como el objeto central y articulador de la pedagogía "por ser el articulador de los demás objetos que se mueven en el campo de la educación: maestro, escuela, aula, niño-alumno, materia o contenido que se enseña, métodos de enseñar o formas de incitar al aprender» (124).

- La didáctica como un campo de formación que define el tipo de profesional en educación y el campo de conocimiento que le es propio a su profesión, en el caso de los profesores de matemáticas, será la didáctica de las matemáticas en su vínculo natural con las matemáticas y las disciplinas afines a la Educación Matemática. Los referentes reflexionan en la didáctica que desarrolla el formador de profesores, que corresponde a una didáctica de la didáctica de las matemáticas.

En estos tres ejes de formación de profesores, se pueden discriminar mejor los elementos para la formación de profesores si se precisan los campos de formación matemática, los tipos de prácticas para aprender a enseñar y ciclos de escolarización en la educación. 
Los ejes estructurantes de la práctica matemática escolar son macro unidades de conocimiento matemático necesarias para el desarrollo de una consciencia matemática básica como ciudadano. Esta última condición es la que determina que Cantidad, Forma y Magnitud, sean consideradas macro unidades.

- Cantidad, Forma y Magnitud, en situaciones deterministas o probabilísticas, encuentran un universo inmediato de exploración, conservación y desarrollo de las poblaciones de la región en el potencial de recursos naturales, a saber, una biodiversidad que representa más del $40 \%$ de las especies vegetales y animales del planeta, un subsuelo que contiene más de la tercera parte de los recursos hídricos renovables de la Tierra y la mayor reserva de tierras cultivables del planeta (Ovalles, 2006), así como la alta diversidad de suelos y la gran variedad de metales en ellos. (71)

- Por una parte, en cantidad, forma y magnitud se encuentran campos de relación con los entornos geográficos y los sistemas de organización social y económica en los que han emergido (Lappan et al. 2006), en particular con los sistemas de organización del tiempo y de las actividades grupales (Bishop, 2005). Por otra parte, en todas las culturas se identifican procesos para contar, comparar, calcular, estimar, medir, construir o temporalizar (Bishop, 1999), los cuales constituyen el ambiente propicio para el desarrollo de estructuras matemáticas (de números, formas, razones, funciones y datos, con sus operaciones, algoritmos y relaciones) que a su vez desarrollan macro-estructuras como la aritmética, la geometría, la estadística y el análisis (Mac Lane, 1986). El ambiente cultural de cada grupo social determina tanto los niveles de desarrollo en las estructuras matemáticas vinculadas a cantidad, forma y magnitud, como el tipo de matemática escolar que en ellos se desarrolla. (110)

Tres prácticas se destacan como fundamentales en la experiencia de aprender a enseñar: diseñar, gestionar y evaluar.

Aunque el referente se consolida en un enunciado, el marco de enunciación que lo acompaña proporciona los elementos que le dan la fuerza realizativa al referente

\section{Referentes para la formación en una Educación Matemática}

Este grupo de referentes considera la educación como el campo profesional del educador matemático y como una gran práctica social que incorpora la acción profesional de todos los educadores. Los referentes son agrupados bajo dos aspectos: i) El regional que reconoce a América Latina y el Caribe como el gran escenario para el desarrollo del campo profesional de la Educación Matemática para poblaciones diversas y como el ambiente natural en el que sucede este tipo de educación como práctica social; ii) El social que reconoce la función social de la Educación Matemática en contextos de diversidad en América Latina y el Caribe. 
Cuadro No. 01. Referentes para formar un educador matemático

\begin{tabular}{|c|c|}
\hline Tipologías de referentes & Número y enunciado del referente \\
\hline \multirow{3}{*}{$\begin{array}{l}\text { América Latina y el Caribe: una fuente de recursos para la } \\
\text { formación del educador matemático }\end{array}$} & $\begin{array}{l}\text { R1: La diversidad geográfica, cultural, poblacio- } \\
\text { nal y lingüística de América Latina y el Caribe } \\
\text { es un recurso para el desarrollo de una expe- } \\
\text { riencia formativa del educador matemático. }\end{array}$ \\
\hline & $\begin{array}{l}\text { R 2: Las condiciones de todas las poblaciones } \\
\text { en América Latina y el Caribe son una fuente } \\
\text { de problemas, preguntas y conflictos que } \\
\text { orientan el desarrollo de políticas para la edu- } \\
\text { cación y la formación de profesores de mate- } \\
\text { máticas. }\end{array}$ \\
\hline & $\begin{array}{l}\text { R3: Las acciones matemáticas de las poblacio- } \\
\text { nes en América Latina y el Caribe se constitu- } \\
\text { yen a partir de las sinergias entre tecnologías } \\
\text { que se producen por la necesaria relación en- } \\
\text { tre poblaciones con tradiciones ancestrales en } \\
\text { el manejo de herramientas y poblaciones que } \\
\text { incorporan tecnologías de punta en sus prácti- } \\
\text { cas profesionales, sociales y familiares. }\end{array}$ \\
\hline \multirow{4}{*}{$\begin{array}{l}\text { La Educación Matemática es un factor de desarrollo social, } \\
\text { cultural y científico de las poblaciones de América Latina }\end{array}$} & $\begin{array}{l}\text { R4: La educación como eje de desarrollo } \\
\text { humano en América Latina y el Caribe se } \\
\text { conforma según las transformaciones que se } \\
\text { dinamizan por relaciones entre tres tipos de } \\
\text { acciones: de asignación de recursos, de acceso } \\
\text { a recursos y de optimización de recursos, para } \\
\text { la educación y para la Educación Matemática } \\
\text { en particular. }\end{array}$ \\
\hline & $\begin{array}{l}\text { R5: El desarrollo de la Educación Matemática } \\
\text { en un país vincula todos los sectores sociales } \\
\text { que lo conforman. }\end{array}$ \\
\hline & $\begin{array}{l}\text { R6: La Educación Matemática prepara al estu- } \\
\text { diante para ser un actor social y político de su } \\
\text { comunidad y de su cultura. }\end{array}$ \\
\hline & $\begin{array}{l}\text { R7: La Educación Matemática promueve y de- } \\
\text { sarrolla los valores de las poblaciones y de su } \\
\text { convivencia en comunidad. }\end{array}$ \\
\hline
\end{tabular}

\section{Referentes para la formación en una pedagogía que acoge las diferencias de las poblaciones}

Este grupo de referentes profundiza en los aspectos pedagógicos en la formación de los docentes de matemáticas, que por una parte tienen relación con la construcción 
de la identidad del profesor, y por otra, con la construcción de una consciencia ética, social, política en el mundo. Se pretende "una práctica educativa en la que ese respeto, que sé que debo tener para con el educando, se realice en lugar de ser negado" (Freire, 1998, citado en León et. al, 2014 p. 91).

Cuadro No. 02. Referentes para la formación pedagógica del profesor

\begin{tabular}{|c|c|}
\hline Tipologías de referentes & Número y enunciado del referente \\
\hline \multirow{3}{*}{$\begin{array}{l}\text { La pedagogía es un factor importante para favorecer el } \\
\text { reconocimiento y coexistencia de la diversidad en los } \\
\text { contextos educativos }\end{array}$} & $\begin{array}{l}\text { R8: La pedagogía reconstruye las relaciones } \\
\text { en la escuela y con la sociedad para hacer } \\
\text { posible la coexistencia con la diversidad. }\end{array}$ \\
\hline & $\begin{array}{l}\text { R9: La pedagogía como generadora de } \\
\text { currículos innovadores y dinámicos que } \\
\text { propicien la formación de docentes de } \\
\text { matemáticas para el trabajo en contextos } \\
\text { de diversidad. }\end{array}$ \\
\hline & $\begin{array}{l}\text { R10: Las comunidades de práctica son una } \\
\text { forma natural de organización de las comu- } \\
\text { nidades educativas en contextos de diver- } \\
\text { sidad, en los que la pedagogía contribuye a } \\
\text { reconocer los diferentes tipos de poblacio- } \\
\text { nes que coexisten en el ecosistema hetero- } \\
\text { géneo del aula. }\end{array}$ \\
\hline \multirow[b]{2}{*}{$\begin{array}{l}\text { La pedagogía, constructora de la identidad del educador } \\
\text { matemático, para la atención de poblaciones en contextos } \\
\text { de diversidad }\end{array}$} & $\begin{array}{l}\text { R11: La pedagogía como constructora de } \\
\text { sentido de la práctica educativa del pro- } \\
\text { fesor de matemáticas que reconoce a los } \\
\text { estudiantes desde sus posibilidades como } \\
\text { sujetos. }\end{array}$ \\
\hline & $\begin{array}{l}\text { R12: La formación pedagógica brinda las } \\
\text { bases conceptuales y las competencias } \\
\text { necesarias para transformar las prácticas } \\
\text { educativas y las concepciones sobre la di- } \\
\text { versidad y las posibilidades del aprendizaje } \\
\text { de las matemáticas en la población sorda, } \\
\text { ciega, indígena o en condición de vulnera- } \\
\text { bilidad económica. }\end{array}$ \\
\hline
\end{tabular}

\section{Referentes para la formación en una didáctica de las matemáticas sin marginaciones}

Este grupo de referentes selecciona de las teorías didácticas vigentes, los fundamentos para una didáctica que le es propia al formador de profesores, se configura una didáctica de la didáctica de las matemáticas, que tiene como objeto de estudio las prácticas de aprender a enseñar las matemáticas y de formar profesores de matemáticas. Los 
aspectos para la agrupación de los referentes destacan experiencias con los objetos de la didáctica de la matemática, experiencias en aprender a enseñar y experiencias en ambientes de aprendizajes.

Cuadro No. 3. Referentes para la formación didáctica del profesor de matemáticas

\begin{tabular}{|c|c|}
\hline Tipologías de referentes & Número y enunciado del referente \\
\hline \multirow{3}{*}{$\begin{array}{c}\text { Las experiencias con los objetos de la didáctica de las } \\
\text { matemáticas son requerimientos para la formación } \\
\text { didáctica de profesores de matemáticas en y para la } \\
\text { diversidad }\end{array}$} & $\begin{array}{l}\text { R13:Experiencias matemáticas y didácticas } \\
\text { que permiten la interacción entre diversos, } \\
\text { dinamizan la formación didáctica para } \\
\text { profesores en situación de diversidad }\end{array}$ \\
\hline & $\begin{array}{l}\text { R14: Cantidad, forma y magnitud estructu- } \\
\text { ran campos de la matemática, de la forma- } \\
\text { ción de profesores y de la organización de } \\
\text { los contenidos escolares }\end{array}$ \\
\hline & $\begin{array}{l}\text { R15:Los tipos de experiencias matemáticas } \\
\text { y didácticas que surgen de la interacción } \\
\text { entre diversos enriquecen y problematizan } \\
\text { la formación de profesores }\end{array}$ \\
\hline \multirow{3}{*}{$\begin{array}{c}\text { Las experiencias en la formación en una didáctica de la } \\
\text { didáctica de las matemáticas, basada en la interacción } \\
\text { entre diversos, reconfiguran la didáctica del formador de } \\
\text { profesores }\end{array}$} & $\begin{array}{l}\text { R16: Experiencias para el diseño, gestión } \\
\text { y evaluación para la interacción entre es- } \\
\text { tudiantes para profesores en situación de } \\
\text { diversidad. }\end{array}$ \\
\hline & $\begin{array}{l}\text { R17: Diseño gestión y evaluación desarro- } \\
\text { llan la identidad del profesor de matemáti- } \\
\text { cas en la diversidad }\end{array}$ \\
\hline & $\begin{array}{l}\text { R18: Diseñar, gestionar y evaluar, son acti- } \\
\text { vidades que posibilitan instaurar procesos } \\
\text { de negociación de significados en aulas de } \\
\text { didáctica de las matemáticas con comuni- } \\
\text { dades de profesores para la diversidad. }\end{array}$ \\
\hline \multirow{3}{*}{$\begin{array}{l}\text { Las experiencias en ambientes de aprendizaje } \\
\text { interculturales y pluritecnológicos son elementos } \\
\text { estructurantes y estructuradores de las experiencias de } \\
\text { aprender la práctica de enseñar las matemáticas }\end{array}$} & $\begin{array}{l}\text { R19: Las experiencias en ambientes de } \\
\text { aprendizaje interculturales y pluritecnoló- } \\
\text { gicos en la Educación Matemática desarro- } \\
\text { llan identidad del profesor de matemáticas } \\
\text { en la diversidad }\end{array}$ \\
\hline & $\begin{array}{l}\text { R20: Participación, interacción, comunica- } \\
\text { ción y mediación semiótica instrumental } \\
\text { renuevan la identidad del estudiante para } \\
\text { profesor de matemáticas. }\end{array}$ \\
\hline & $\begin{array}{l}\text { R21: Los ambientes interculturales y pluri- } \\
\text { tecnológicos dinamizan el desarrollo del } \\
\text { conocimiento matemático de poblaciones. }\end{array}$ \\
\hline
\end{tabular}




\section{Compromiso social con la acción formativa de profesores y el efecto de los referentes}

El último capítulo de los referentes proporciona orientación para el uso de los referentes por diferentes actores del sistema educativo, en este capítulo se destacan tres tipos de actores que por su responsabilidad en la educación están llamados a incorporar los referentes en sus prácticas profesionales:

- Instituciones que generan política educativa (ministerios, secretarías): Los referentes curriculares pueden ser usados en la evaluación, el diseño o la experimentación de programas de formación de profesores de matemáticas que se comprometan con acoger a la diversidad ( $\left.\mathrm{R}_{1}, \mathrm{R}_{2}\right)$; o también para obtener la concurrencia de todos los actores sociales involucrados en los procesos educativos $\left(\mathrm{R}_{3}, \mathrm{R}_{5}\right)$

- Instituciones formadoras de profesores (universidades, institutos de educación, normales, facultades de educación y de ingeniería): Los referentes pueden ser usados para crear, desarrollar, reformular y orientar, currículos de formación de profesores de matemáticas (R6, R7); también para promover experiencias formativas que reconozcan la diversidad en los futuros profesores con prácticas pedagógicas en el mismo sentido (R14, R17).

- Formadores de profesores de matemáticas, estudiantes para profesor de matemáticas y profesores de matemáticas: Los referentes pueden ser usados para promover tres tipos de experiencias para la formación: con los objetos de la matemática y su didáctica, con los objetos de la didáctica de la didáctica de las matemáticas y en ambientes de aprendizaje pluritecnológicos y multiculturales con una propuesta de dos enfoques en la formación con tecnologías compatibles con estas experiencias (R6, R11; R13.14.15 y 20); en la organización de espacios académicos para el desarrollo de diversas experiencias formativas en ambientes de aprendizaje (R13, R14, R15, R19).

La experiencia de validación de los referentes en la construcción de objetos virtuales de aprendizaje y la observación del uso de éstos últimos, en escenarios naturales de formación de profesores de matemáticas, con una valoración por encima de 9o, de un total de 100 posible, en profesores, de 85 en observadores y de 80 en estudiantes, evidenció que los rasgos fundamentales de los referentes fueron identificados por los profesores, por los observadores y por los estudiantes.

Este proceso hace de la obra "Referentes curriculares con incorporación tecnológica para la formación del profesorado de matemáticas en y para la diversidad" un instrumento eficaz para la formación de profesores de matemáticas. 


\section{Lista de referencias}

Ball, D., Thames, M. y Phelps, G. (2008). Content knowledge for teaching: What makes it special? Journal of Teacher Education, 59(5), 389-407.

CEPAL. (2014). Panorama Social de América Latina. Santiago de Chile: Naciones Unidas.

Freire, P. (1998). Cultural Action for Freedom. Harvard Educational Review, 68(4), 471-521.

León, O., Bonilla, M. B., Romero, J., Gil, D. C., Avila, C., Bacca, J., Aleja, M. (2014). Referentes curriculares con incorporación de tecnologías para la formación del profesorado de matemáticas en y para la diversidad (Segunda ed.). (O. L. León, Ed.) Bogotá, Colombia: Universidad Distrital Francisco José de Caldas.

León, O., Bonilla, M., Romero, J., Gil, D., Correal, M., Avila, C., Bacca, J., Cavanzo, A., Guevara, C., Saiz, M., García, R., Saiz, E., Rojas, N., Peralta, M., Flores, W., \& Márquez, A. (2013). Referentes curriculares con incorporación de tecnologías para la formación de profesorado de matemáticas. México D.F.: Universidad Pedagógica Nacional.

MacLane, S. (1986). Mathematics form and function. New York: Springer- Verlag.

Ovalles, F. (2006). Manejo Sustentable de los Recursos Naturales en América Latina y el Caribe: Oportunidades y Desafíos de Investigación y Desarrollo Tecnológico para la Cooperación. Maracaibo: INIA-CENIAP.

Serce (2008). Los aprendizajes de los estudiantes de América Latina y el Caribe. Santiago de Chile: UNESCO-LLECE.

Shulman, S. (1986). ose who understand: Knowledge growth in teaching. Educational Researcher, 15, 4-14.

Vasco, C., Martínez, A. y Vasco, E. (2008). Educación; Pedagogía y Didáctica una Perspectiva Epistemológica. En G. Hoyos (Ed.), Filosofía de la Educación (pp. 99-127). Bogotá: Trotta. 